Neubewertung der Dienste für Migrant(inn)en und Asylbewerber(innen) ohne Papiere.

Die Mitgliedstaaten sollten erwägen, wie sich die Lage von Asylbewerber(inne)n und Migrant(inn)en ohne offiziellen Aufenthaltsstatus verbessern lässt. Die Mitgliedstaaten sollten sicherstellen, dass für diese Gruppe die Gesundheitsversorgung gesichert ist. Diese Verpflichtung ergibt sich nach Auffassung des djb bereits aus dem Grundrechts- und Menschenrechtsschutz dieser Gruppen.

\section{Durchführung spezifischer Folgenabschätzungen großer sozialer und gesundheitspolitischer Veränderungen mit Blick auf die Situation der von sozialer Ausgrenzung bedrohten Menschen.}

Wenn die Mitgliedstaaten die Systeme zur Finanzierung der Gesundheitsversorgung einschließlich der Kostenteilungsregelungen ändern, sollte bei der routinemäßigen Datenerhebung verletzlichen Gruppen besondere Aufmerksamkeit geschenkt werden, um sicherzustellen, dass politische Maßnahmen zur Vermeidung von Ausgrenzung tatsächlich greifen. Es bedarf einer Kultur des Monitoring und der Programmbewertung, um bedeutende Lücken in den Forschungs- und Informationssystemen zu schließen.

Die Mitgliedstaaten müssen stärker darauf achten, dass die Gründe dafür, dass es einigen Menschen nicht gelingt, einen regulären Versicherungsstatus zu erlangen, systematisch erforscht werden. Dies sollte für Länder unternommen werden, wo gewisse verletzliche Gruppen Gefahr laufen, vom regulären Gesundheitsversorgungsschutz ausgegrenzt zu werden.

\section{Größere Berücksichtigung der für die verletzlichen Gruppen bestehenden Zugangsbarrieren im politischen Prozess der Europäischen Union.}

$\mathrm{Zu}$ den vorrangigen Themen für einen breiter angelegten europäischen Austausch zählen Erschwinglichkeit der Gesundheitsversorgung für verletzliche Gruppen, Stärkung der Gesundheitskompetenz im Allgemeinen und Stärkung der Patient(inn)en.

\section{Referenzen}

Europäische Kommission (2006). Gemeinsamer Bericht über Sozialschutz und soziale Eingliederung 2006, Brüssel.

Rat der Europäischen Union (2006). Schlussfolgerungen des Rates zum Thema gemeinsame Werte und Prinzipien in den europäischen Gesundheitssystemen, 2733. Tagung des Rates der Europäischen Union (Beschäftigung, Sozialpolitik, Gesundheit und Verbraucherschutz), Luxemburg 1-2 Juni 2006.

European Commission, Quality in and Equality of Access to Healthcare Services, März 2008.

\title{
Exklusion/Inklusion aus deutscher Perspektive: Nationaler Integrationsplan
}

\section{Sonka Gerdes}

Referentin im Bundesministerium für Familie, Senioren, Frauen und Jugend, Berlin

„Wir wollen miteinander und nicht übereinander sprechen.“ Dies sagte Bundeskanzlerin Angela Merkel beim ersten Integrationsgipfel am 14. Juli 2006, zu dem sie vor allem Migran$\mathrm{t}$ (inn)en ins Kanzleramt eingeladen hatte.

In der Folge wurde innerhalb eines Jahres in zehn Arbeitsgruppen mit einem breiten Spektrum an Vertreter(inne)n staatlicher und nichtstaatlicher Organisationen der Nationale Integrationsplan (NIP) erarbeitet. Teilgenommen haben Vertreter(innen) von Bund, Ländern und Kommunen, Arbeitgeber und Gewerkschaften, Kirchen und Religionsgemeinschaften, Wohlfahrtsverbände und Stiftungen, Medien, Kulturschaffende, Sportverbände und Migrant(inn)en sowie ihre Organisationen. Erstmals wurde so - organisiert von der Staatsministerin für Integration, Prof. Dr. Maria Böhmer - eine gemeinsame Plattform für Integrationspolitik in Deutschland geschaffen, die die Aktivitäten von Bund, Ländern, Kommunen und nichtstaatlichen Akteuren vereinigt.
Die Arbeitsgruppen haben zu den Themen Integrationskurse, Sprachförderung, Bildung und Ausbildung, Lebenssituation von Frauen und Mädchen verbessern, Integration vor Ort, Kultur und Integration, Integration durch Sport, Medien, bürgerschaftliches Engagement und Wissenschaft gearbeitet.

Der daraus entstandene Nationale Integrationsplan (NIP) enthält Erklärungen von Bund, Ländern und kommunalen Spitzenverbänden für eine nachhaltige Integrationspolitik sowie die Ergebnisse der Arbeitsgruppen. Er enthält rund 400 Selbstverpflichtungen der teilnehmenden Organisationen. Im Juli 2007 wurde er der Öffentlichkeit vorgestellt.

Am 6. November 2008 wurde mit dem Ersten Fortschrittsbericht die erste Zwischenbilanz zur Umsetzung präsentiert, in dem die zahlreichen Aktivitäten und Weiterentwicklungen dokumentiert werden. Von den Maßnahmen des Bundes seien folgende genannt:

- Die Integrationskurse wurden qualitativ und quantitativ aufgewertet, was zu einer deutlichen Erhöhung der Teilnehmerzahlen - insbesondere bei den freiwilligen Teilnehme$\mathrm{r}$ (inne) $\mathrm{n}$ - geführt hat.

- Beim Bildungsgipfel haben die Regierungschefs von Bund und Ländern eine „Qualifizierungsinitiative für Deutsch- 
land" beschlossen, mit der sie ein deutliches Signal für eine höhere Bildungsbeteiligung und verbesserte Aufstiegsmöglichkeiten durch Bildung gerade auch für Migrant(inn)en gesetzt haben.

- Der Zugang ausländischer Jugendlicher und junger Erwachsener zu BAföG und Berufsausbildungsbeihilfe wurde durch eine Gesetzesnovelle deutlich erleichtert.

\section{Die Rolle der Migrantinnen im Nationalen Inte- grationsplan}

Frauen war im Rahmen dieses Prozesses besondere Aufmerksamkeit zu schenken. In ihrem Alltag verdichten sich oft die Notwendigkeiten und Erfordernisse, aber auch die Spielräume und Handlungsoptionen des durch die Migration veränderten Lebens. Sie sind es, die einerseits einen Großteil der Belastungen zu tragen haben und andererseits wichtige Weichenstellungen für den eigenen Integrationsprozess und den ihrer Kinder vornehmen können.

\section{Die Bundesregierung hat mit dem Nationalen Integra- tionsplan auf die zunehmende sprachliche und kultu- relle Vielfalt der Bevölkerung in Deutschland reagiert und damit einen Paradigmenwechsel in der Integrationspolitik eingeleitet.}

Die Potenziale der Migrantinnen müssen daher genutzt werden. Ohne angemessene Berücksichtigung der Rolle von Frauen und Mädchen, ihrer besonderen Probleme und ihrer spezifischen Bedürfnisse kann Integration nicht gelingen.

Die Situation von Frauen und Mädchen war dementsprechend ein Schwerpunktthema bei der Erarbeitung des NIP. In allen Themenfeldern sollten geschlechtsspezifische Aspekte gezielt berücksichtigt werden. Zusätzlich hat die Arbeitsgruppe 4 des NIP zum Thema „Lebenssituation von Frauen und Mädchen verbessern, Gleichberechtigung verwirklichen" gearbeitet.

In den letzten Jahren sind Migrantinnenorganisationen entstanden, um Frauen und Familien mit Migrationshintergrund zu beraten, weiterzubilden oder anderweitig zu unterstützen. Diese zivilgesellschaftlichen Organisationen waren gut in der Arbeitsgruppe vertreten. Von ihnen sind zahlreiche Selbstverpflichtungen in den NIP eingebracht worden.

Teilgenommen haben zum Beispiel das Begegnungs- und Fortbildungszentrum muslimischer Frauen e.V. in Köln, der Bundesverband der Mi- grantinnen in Deutschland und Papatya - anonyme Kriseneinrichtung für Mädchen und junge Frauen mit Migrationshintergrund aus Berlin.

\section{Inhalt der Arbeitsgruppe zur Lebenssituation von Frauen und Mädchen}

Die Arbeitsgruppe zur Lebenssituation von Frauen und Mädchen unterteilte sich in zwei Unterarbeitsgruppen. Die erste Unterarbeitsgruppe „Integration durch Recht" beschäftigte sich vor allem mit der Bekämpfung von Gewalt gegen Migrantinnen, wobei ein Schwerpunkt das Thema Zwangsverheiratung darstellte. Daneben stellte die Arbeitsgruppe die gesellschaftliche und politische Teilhabe von Frauen und Mädchen in den Mittelpunkt. Hierzu gehört neben der Organisation und Vernetzung von Migrantinnen und ihrer Interessenvertretungen auch die Verbesserung des Zugangs von Migrantinnen zu Ausbildung und Beruf, denn Teilhabe am Arbeitsmarkt und Teilhabe an der Gesellschaft sind eng miteinander verknüpft.

Die „sozialen Rechte“, die Gegenstand dieses Podiums sind, waren Gegenstand der zweiten Unterarbeitsgruppe. Sie befasste sich mit den Themen „Stärkung der Migrantinnen in Familie und sozialem Umfeld, Sexualaufklärung, Gesundheit und Altenhilfe". Eine eigene Arbeitsgruppe zum Thema Gesundheit und Alter gab es bei der Erstellung des NIP nicht. Diese Themen wurden ausschließlich in dieser Unterarbeitsgruppe bearbeitet. Ihre Relevanz wurde während der Arbeit aber sehr deutlich. Im Fortschrittsbericht 2008 gibt es im Beitrag der Länder eigene Kapitel zu Gesundheit und Alter.

Einigkeit bestand in der Arbeitsgruppe, dass die Vielfalt der Frauen und Mädchen mit Migrationshintergrund, ihre unterschiedlichen Lebenslagen und auch die Pluralität ihrer Lebensentwürfe in der Öffentlichkeit nicht wahrgenommen werden. In der Konsequenz werden ihre Ressourcen und Potenziale wenig gesehen und Maßnahmen werden am Bedarf vorbei konzipiert. Die Integrationspolitik muss daher einer Stereotypisierung des Bildes von Migrantinnen in der Gesellschaft entgegenwirken. Passende Angebote werden in der Praxis insbesondere von Migrantinnenselbstorganisationen oder in Kooperation mit ihnen erbracht.

Zum Thema Gesundheit wurde festgestellt, dass das präventive Regelangebot der Gesetzlichen Krankenkassen oft noch wenig von Migrantinnen wahrgenommen wird. Trotz grundsätzlicher Zugangsoffenheit zum Angebot der Vorsorgeleistungen sind Migrantinnen beispielsweise bei der Inanspruchnahme zahnmedizinischer Präventivmaßnahmen oder bei der Vorsorge in der Schwangerschaft deutlich unterrepräsentiert. Dies zeigt, 
dass grundsätzlich gleiche Nutzungsrechte noch keine gleiche Nutzungseffektivität garantieren.

Immens wichtig ist - auch bei der sensiblen Sexualaufklärung - eine angemessene Sprache sowie Kultursensibilität.

Als Selbstverpflichtung zum Thema Gesundheit wurde unter anderem von der Bundeszentrale für gesundheitliche Aufklärung (BzGA) eingebracht, dass die Online-Datenbank „Frauengesundheit und Gesundheitsförderung“ das Thema Migration und Gesundheit unter verschiedenen Aspekten aufgreifen wird. Das Bundesamt für Migration und Flüchtlinge will zu einer geschlechtsspezifischen und kultursensiblen Gesundheitsaufklärung im Rahmen der bundeseinheitlichen Integrationskurse, des Integrationsprogramms und der Migrationserstberatung beitragen. Die Mitgliedsverbände der Bundesarbeitsgemeinschaft der Freien Wohlfahrtpflege sichern zu, den Prozess der interkulturellen Öffnung in ihren Einrichtungen und Diensten umzusetzen.

Im Fortschrittsbericht 2008 werden eine Reihe von guten Beispielen aus den Ländern genannt. So gibt es im Saarland eine Internetdatenbank „Migration und Gesundheit“, die die Teilhabe von Migrant(inn)en an einer adäquaten Versorgung sicherstellen soll, vor allem durch die Darstellung der Gesundheitsdienste, die über fremdsprachliche Kompetenzen bzw. über Dolmetscherdienste verfügen. In Berlin laufen schon seit langem Projekte - wie z.B. das Modellprojekt „Gesund sind wir stark“ -, in denen Fachleute aus bestehenden lokalen Netzwerken zu Gesundheitstrainern und Mütter und Väter zu Gesundheitsmentoren ausgebildet werden. In NordrheinWestfalen gibt es im Rahmen der Früherkennung von Brustkrebs Seminare für türkische Frauen, die sprachliche und soziokulturelle Besonderheiten berücksichtigen.

Das Thema Integration älterer Migrantinnen wurde insbesondere unter dem Blickwinkel der interkulturellen Öffnung bestehender Angebote der Altenhilfe und dem Schaffen von Einrichtungen für bestimmte Zielgruppen von Migrant(inn)en diskutiert.

Anders als von Politik und Gesellschaft angenommen, ist die Rückkehr ins Herkunftsland für die meisten älteren Migrantinnen keine Alternative mehr. Da Migrantinnen der ersten Zuwanderergeneration vielfach erst jetzt ins Rentenalter kommen, werden ihre Lebenslagen und Bedarfe zudem erst sukzessive wahrgenommen. Breitangelegte, bedarfsgerechte Angebote fehlen bisher.

Dies zeigt sich zum Beispiel beim Thema Demenz. Die derzeitigen Begutachtungsverfahren sind teilweise bei demenzbedingter degenerierender Sprachkompetenz für Migrant(inn) en nicht geeignet. Kultursensible Unterstützungsangebote für pflegende Angehörige gibt es kaum.

Ältere Migrant(inn)en finden häufig keinen Zugang zu Angeboten für Senior(inn)en, zu Pflegediensten und -einrichtungen, obwohl die Angebote auch ihnen offenstehen. Bestehende Angebote sind zudem oft nicht geschlechterdifferenziert. Die gezielte Information und Förderung kultursensibler Arbeitsweisen in Seniorenarbeit und Pflege müssen daher verbessert werden.
Zur Thematik Alter gab es im NIP unter anderem folgende Selbstverpflichtungen: Die Mitgliedsverbände der Bundesarbeitsgemeinschaft der Freien Wohlfahrtspflege e.V. wollen die interkulturelle Öffnung von Diensten und Einrichtungen der Altenpflege und Altenarbeit unter Einbeziehung von Migrantenselbstorganisationen voranbringen. Auch wollen sie vermehrt junge Migrant(inn)en für pflegerische Berufe gewinnen. Der Zentralrat der Muslime will zweisprachige Informationsblätter/Info-Mails unter anderem zur Situation älterer Migran$\mathrm{t}$ (inn)en erstellen.

Im Fortschrittsbericht zum NIP bestätigen alle Bundesländer, dass die Förderung kultursensibler Arbeitsweisen in der Seniorenarbeit und Pflege an Bedeutung gewinnt. Neben einer Vielzahl von Informationsveranstaltungen, Schulungen, Fortund Weiterbildungen für Mitarbeiter(innen) zu Lebenssituation und Altersbildern von Migrant(inn)en wird die interkulturelle Öffnung der Altenpflege in einigen Ländern durch Modellprojekte unterstützt. In Bremen erfolgt die Förderung kultursensibler Arbeitsweisen in der Seniorenarbeit unter anderem über ein umfassendes Gesundheitsförderkonzept und die Vernetzung von Trägern unterschiedlicher Angebotsformen der Altenhilfe und Pflege. In Hessen wurde das Modellprojekt „Kultursensible Altenpflege“ durchgeführt, um mehr Migrant(inn)en für den Altenpflegeberuf zu begeistern und interkulturelle Elemente in der Regelausbildung zu verankern.

Wichtig ist in diesem Zusammenhang auch das Pflege-Weiterentwicklungsgesetz (Gesetz zur strukturellen Weiterentwicklung der Pflegeversicherung), das am 1. Juli 2008 in Kraft getreten ist. Es schreibt erstmals fest, dass neben geschlechtsspezifischen Bedarfen auch den Bedürfnissen nach einer kultursensiblen Pflege nach Möglichkeit Rechnung getragen werden soll.

Die Arbeitsgruppe stellte zum Thema Gesundheit abschließend Folgendes fest:

Die Verbesserung der gesundheitlichen Versorgung von Migrantinnen erfordert

- eine interkulturelle Regelversorgung,

- bedarfsorientierte Angebote,

- eine interdisziplinäre Vernetzung,

- die Erschließung adäquater Zugangswege,

- die Sicherung der Datenbasis.

Dies gilt entsprechend auch für die Verbesserung der Angebote für ältere Migrant(inn)en.

Insgesamt hat sich gezeigt, dass insbesondere die Themen Gesundheit und Alter „Zukunftsthemen“ sind, denen im weiteren Prozess des NIP besondere Aufmerksamkeit gewidmet werden sollte.

\section{Wie steht es mit den „sozialen Rechten“ im Integrations- gipfelprozess?}

Migrantinnen mit einem sicheren Aufenthaltsstatus stehen zumeist die gleichen sozialen Rechte zu - zum Beispiel nach dem Sozialgesetzbuch - wie deutschen Staatsbürgerinnen. Die sozialen Rechte und Ansprüche auf Sozialleistungen von Migrantinnen mit unsicherem Aufenthaltstitel, beispielsweise Flücht- 
lingsfrauen mit einem unsicheren humanitären Aufenthaltstitel, sind hingegen eingeschränkt, obwohl diese oft gerade einer besonderen Unterstützung bedürfen. Besonders prekär ist die Lage der Migrantinnen „ohne Papiere“.

Die Arbeitsgruppen im Integrationsgipfelprozess hatten die Aufgabe, eine Bestandsaufnahme mit Zielbestimmungen zu erstellen und dann konkrete Selbstverpflichtungen zu vereinbaren. Vorschläge für Rechtsänderungen waren grundsätzlich nicht Aufgabe der Arbeitsgruppen. Dennoch hatten rechtliche Fragen eine Relevanz und waren gerade auch in der Arbeitsgruppe zur Lebenssituation der Frauen immer wieder Thema. So wurden Verbesserungen für Betroffene von Gewalt - insbesondere für von Zwangsverheiratungen Betroffene - in dem parallel zum Integrationsgipfelprozess laufenden Gesetzgebungsverfahren des 2. Änderungsgesetzes zum Zuwanderungsgesetz gefordert. Von einigen Arbeitsgruppenmitgliedern wurde ein Widerspruch des Integrationsgipfelprozesses „auf Augenhöhe“ zu den geplanten Restriktionen im Aufenthaltsrecht gesehen und formuliert. Dieser parallele Gesetzgebungsprozess hatte auch zur Folge, dass einige türkische Verbände dem 2. Integrationsgipfel 2007 fernblieben, weil sie die geplante Änderung, Deutschkenntnisse vor Einreise beim Familiennachzug zu verlangen, als ungerechtfertigte Benachteiligung sahen.

Es ist in der Arbeitsgruppe zur Lebenssituation der Frauen deutlich geworden, dass die Information der Frauen mit Migrationshintergrund über die bestehenden Rechte wichtig ist. Denn Kenntnis der eigenen Rechte ist die Voraussetzung für ein wirksames „Empowerment“ und eine verbesserte Nutzungseffektivität bestehender Angebote.

Dementsprechend enthält der NIP verschiedene Selbstverpflichtungen zu Aufklärungsmaßnahmen über bestehende Rechte, zum Beispiel von der Bundesarbeitsgemeinschaft der Freien Wohlfahrtspflege e.V., der Frauenhauskoordinierung, dem Begegnungs- und Fortbildungszentrum muslimischer Frauen e.V. und dem Bundesverband der Migrantinnen.

\section{Fehlende Daten/Integrationsmonitoring}

Deutlich geworden ist insgesamt, dass vielfach Daten fehlen: Nicht nur zu Menschen mit Migrationshintergrund allgemein, sondern auch aufgeschlüsselt nach Geschlecht. Nur die genaue Kenntnis der vielfältigen Lebenslagen ermöglicht aber passgenaue politische Maßnahmen. Um hier Abhilfe zu schaffen, hat das Bundesministerium für Familien, Senioren, Frauen und Jugend eine Sonderauswertung des Mikrozensus $2005 \mathrm{zu}$ Geschlecht und Migration in Auftrag gegeben, die bereits vorliegt. In anderen Feldern müssen gezielt Daten erhoben werden. So hat sich die Bundesregierung verpflichtet, die Datenlage zu Zwangsverheiratungen zu verbessern, um diese bekämpfen und Betroffene effektiv unterstützen zu können.

Ergebnis des Integrationsgipfelprozesses ist auch, dass der Bund zukünftig im Rahmen eines Integrationsmonitorings Integration im Zeitverlauf beobachtet und den Erfolg von Integrationsmaßnahmen überprüft. Inzwischen sind 100 Indikatoren zu allen relevanten Feldern der Integration innerhalb der Bundesregierung und mit Expert(inn)en abgestimmt. Anhand der vorliegenden Datensätze wurde der erste Integrationsindikatorenbericht vorgelegt und im Juni 2009 vom Kabinett zur Kenntnis genommen. In ihm wurden geschlechtsspezifische Daten so weit wie möglich berücksichtigt. Der Bericht enthält einige vertiefte Analysen, in denen er differenziert sozialstrukturelle und migrationsbedingte Einflussfaktoren in den Blick nimmt.

Hierbei wurde beispielsweise im Bereich Bildung deutlich, dass migrationsspezifische Merkmale durch sozialstrukturelle Faktoren, den Sprachgebrauch im Haushalt und die Bildungserwartung der Eltern erklärt werden können: Die weniger erfolgreichen Schulkarrieren von Jugendlichen mit Migrationshintergrund können demnach teilweise darauf zurückgeführt werden, dass im Haushalt nicht deutsch gesprochen wird und die Eltern ein geringes Bildungsniveau sowie eine geringe Bildungsorientierung aufweisen.

Der erste „Probelauf“ des Monitorings hat gezeigt, dass das Indikatorenset einer Bearbeitung und Straffung bedarf. Schwierigkeiten bestehen auch, weil bisher nur teilweise die aussagekräftigen Daten zum Migrationshintergrund vorliegen. Viele Datensätze beziehen sich (noch) auf die Staatsangehörigkeit. Ein überarbeitetes Indikatorenset soll die Grundlage des zweiten Integrationsindikatorenberichts im Jahr 2011 sein.

\section{Bewertung des Integrationsgipfelprozesses}

Die Bundesregierung hat mit dem NIP auf die zunehmende sprachliche und kulturelle Vielfalt der Bevölkerung in Deutschland reagiert und damit - so kann man sagen - einen Paradigmenwechsel in der deutschen Integrationspolitik eingeleitet.

Integrationspolitik ist damit auf allen Ebenen zu einem Querschnittsthema geworden.

Auch international ist dieser Prozess, an dem alle gesellschaftlichen Kräfte und insbesondere die Migrant(inn)en teilnehmen, vorbildlich und auch einmalig. Flankiert wird der Prozess im Übrigen von Integrationsplänen auf Landesebene (z.B. in Nordrhein-Westfalen und Baden-Württemberg) und auch von kommunalen Integrationsplänen.

Die Bestandsaufnahmen der unterschiedlichen Arbeitsgruppen sind aufgrund der breit gefächerten Zusammensetzung der Gruppen wichtige Dokumente. Viele Projekte, die in den NIP eingegangen sind, mögen bereits geplant gewesen sein. Es sind aber auch viele neue Projekte aufgenommen worden, deren Durchsetzung durch die Arbeiten am NIP befördert oder die deswegen erst entwickelt wurden. So gibt es zum Beispiel im 2. Aktionsplan zur Bekämpfung von Gewalt gegen Frauen einen Schwerpunkt zum Thema Migration, der sicherlich durch die Arbeiten am NIP verstärkt werden konnte. Durch die Beobachtung und Evaluierung der Umsetzung ist eine Nachhaltigkeit des Prozesses gewährleistet.

Wichtig ist auch, dass durch die Arbeit in den Arbeitsgruppen vielfältige Kontakte entstanden sind, insbesondere auch zu den Vertreter(inne)n der Migrant(inn)en, die nun fruchtbar weiter eingesetzt werden. 